ISSN 1855-3966 (printed edn.), ISSN 1855-3974 (electronic edn.)

ARS MATHEMATICA CONTEMPORANEA 20 (2021) 233-241

https://doi.org/10.26493/1855-3974.2284.aeb

(Also available at http://amc-journal.eu)

\title{
Closed formulas for the total Roman domination number of lexicographic product graphs
}

\author{
Abel Cabrera Martínez (D), Juan Alberto Rodríguez-Velázquez \\ Universitat Rovira i Virgili, Departament d'Enginyeria Informàtica i Matemàtiques, \\ Av. Països Catalans 26, 43007 Tarragona, Spain
}

Received 19 March 2020, accepted 6 January 2021, published online 6 November 2021

\section{Abstract}

Let $G$ be a graph with no isolated vertex and $f: V(G) \rightarrow\{0,1,2\}$ a function. Let $V_{i}=\{x \in V(G): f(x)=i\}$ for every $i \in\{0,1,2\}$. We say that $f$ is a total Roman dominating function on $G$ if every vertex in $V_{0}$ is adjacent to at least one vertex in $V_{2}$ and the subgraph induced by $V_{1} \cup V_{2}$ has no isolated vertex. The weight of $f$ is $\omega(f)=$ $\sum_{v \in V(G)} f(v)$. The minimum weight among all total Roman dominating functions on $G$ is the total Roman domination number of $G$, denoted by $\gamma_{t R}(G)$. It is known that the general problem of computing $\gamma_{t R}(G)$ is NP-hard. In this paper, we show that if $G$ is a graph with no isolated vertex and $H$ is a nontrivial graph, then the total Roman domination number of the lexicographic product graph $G \circ H$ is given by

$$
\gamma_{t R}(G \circ H)= \begin{cases}2 \gamma_{t}(G) & \text { if } \gamma(H) \geq 2, \\ \xi(G) & \text { if } \gamma(H)=1,\end{cases}
$$

where $\gamma(H)$ is the domination number of $H, \gamma_{t}(G)$ is the total domination number of $G$ and $\xi(G)$ is a domination parameter defined on $G$.

Keywords: Total Roman domination, total domination, lexicographic product graph.

Math. Subj. Class. (2020): 05C69, 05C76

\section{Introduction}

Let $G$ be a graph with no isolated vertex and $f: V(G) \rightarrow\{0,1,2\}$ a function. Let $V_{i}=$ $\{x \in V(G): f(x)=i\}$ for every $i \in\{0,1,2\}$. We will identify $f$ with the partition of

E-mail addresses: abel.cabrera@urv.cat (Abel Cabrera Martínez), juanalberto.rodriguez@urv.cat (Juan Alberto Rodríguez-Velázquez)

(a)(i) This work is licensed under https://creativecommons.org/licenses/by/4.0/ 
$V(G)$ induced by $f$ and write $f\left(V_{0}, V_{1}, V_{2}\right)$. The weight of $f$ is defined to be

$$
\omega(f)=f(V(G))=\sum_{v \in V(G)} f(v)=\left|V_{1}\right|+2\left|V_{2}\right| .
$$

A function $f\left(V_{0}, V_{1}, V_{2}\right)$ is said to be total Roman dominating function on $G$ if every vertex in $V_{0}$ is adjacent to at least one vertex in $V_{2}$ and the subgraph induced by $V_{1} \cup V_{2}$ has no isolated vertex [17]. The minimum weight among all total Roman dominating functions on $G$ is the total Roman domination number of $G$, denoted by $\gamma_{t R}(G)$. In this article, we continue the study initiated in [5] on the total Roman domination number of lexicographic product graphs. In particular, we give closed formulas for the total Roman domination number of lexicographic product graphs.

Let $G$ and $H$ be two graphs. The lexicographic product of $G$ and $H$ is the graph $G \circ H$ whose vertex set is $V(G \circ H)=V(G) \times V(H)$ and $(u, v)(x, y) \in E(G \circ H)$ if and only if $u x \in E(G)$ or $u=x$ and $v y \in E(H)$. Notice that for any $u \in V(G)$ the subgraph of $G \circ H$ induced by $\{u\} \times V(H)$ is isomorphic to $H$. For simplicity, we will denote this subgraph by $H_{u}$.

For a basic introduction to the lexicographic product of two graphs we suggest the books [7, 12]. One of the main problems in the study of $G \circ H$ consists of finding exact values or tight bounds for specific parameters of these graphs and express them in terms of known invariants of $G$ and $H$. In particular, we cite the following works on domination theory of lexicographic product graphs: (total) domination [14, 18, 19, 21], Roman domination [14], weak Roman domination [20], rainbow domination [15], super domination [6], doubly connected domination [2], secure domination [13], double domination [3] and total Roman domination [5].

We assume that the reader is familiar with the basic concepts and terminology of domination in graph. If this is not the case, we suggest the textbooks [8, 9, 11]. In particular, we use the standard notation $\gamma(G)$ and $\gamma_{t}(G)$ for the domination number and the total domination number of a graph $G$, respectively. Throughout the paper, $N(v)$ will denote the set of neighbours or open neighbourhood of $v$ in $G$. The closed neighbourhood of $v$, denoted by $N[v]$, equals $N(v) \cup\{v\}$. A vertex $v \in V(G)$ such that $N[v]=V(G)$ is said to be a universal vertex. For the remainder of the paper, definitions will be introduced whenever a concept is needed.

\section{The case where $\gamma(H) \geq 2$}

The next theorem merges two results obtained in [14] and [21].

Theorem 2.1 ([14] and [21]). For any graph $G$ with no isolated vertex and any nontrivial graph $H$,

$$
\gamma(G \circ H)= \begin{cases}\gamma(G), & \text { if } \gamma(H)=1 \\ \gamma_{t}(G), & \text { if } \gamma(H) \geq 2\end{cases}
$$

Below we present two theorems that complete the tools we need to deduce our first result.

Theorem 2.2 ([1]). For any graph $G$ with no isolated vertex,

$$
2 \gamma(G) \leq \gamma_{t R}(G) \leq \min \left\{2 \gamma_{t}(G), 3 \gamma(G)\right\}
$$


Theorem 2.3 ([4]). For any graph $G$ with no isolated vertex and any nontrivial graph $H$,

$$
\gamma_{t}(G \circ H)=\gamma_{t}(G)
$$

From the results above we deduce the following main theorem.

Theorem 2.4. For any graph $G$ with no isolated vertex and any graph $H$ with $\gamma(H) \geq 2$,

$$
\gamma_{t R}(G \circ H)=2 \gamma_{t}(G)
$$

Proof. The result immediately follows by applying Theorems 2.1, 2.3 and 2.2, in this order, i.e., $2 \gamma_{t}(G)=2 \gamma(G \circ H) \leq \gamma_{t R}(G \circ H) \leq 2 \gamma_{t}(G \circ H)=2 \gamma_{t}(G)$.

Notice that, since the general optimization problem of finding the total domination number of a graph is NP-hard [16], by Theorem 2.4 we can conclude that the problem of finding the total Roman domination number is NP-hard. Even so, we would like to point out that there are several families of graphs for which the total domination number can be found in polynomial time [10].

\section{The case where $\gamma(H)=1$}

The following two lemmas are the main tools in this section.

Lemma 3.1. Let $G$ be a graph with no isolated vertex. For any nontrivial graph $H$ with $\gamma(H)=1$, there exists a $\gamma_{t R}(G \circ H)$-function $f$ satisfying the following two conditions.

(i) $f\left(V\left(H_{u}\right)\right) \leq 2$ for every $u \in V(G)$.

(ii) If $f\left(V\left(H_{u}\right)\right)=2$, then $f(u, v)=2$ for some universal vertex $v$ of $H$.

Proof. Given a TRDF $f$ on $G \circ H$, we define the set $R_{f}=\left\{x \in V(G): f\left(V\left(H_{x}\right)\right) \geq 3\right\}$. Let $f$ be a $\gamma_{t R}(G \circ H)$-function such that $\left|R_{f}\right|$ is minimum among all $\gamma_{t R}(G \circ H)$-functions. Let $v \in V(H)$ be a universal vertex and suppose that there exists $u \in R_{f}$. We differentiate the following two cases.

Case 1. There exists $u^{\prime} \in N(u)$ such that $f\left(V\left(H_{u^{\prime}}\right)\right) \geq 1$. Let $f^{\prime}$ be the function defined by $f^{\prime}\left(V\left(H_{u}\right)\right)=f^{\prime}(u, v)=2$ and $f^{\prime}(x, y)=f(x, y)$ for every $x \in V(G) \backslash\{u\}$. It is readily seen that $f^{\prime}$ is a $\gamma_{t R}(G \circ H)$-function with $\left|R_{f^{\prime}}\right|<\left|R_{f}\right|$, which is a contradiction.

Case 2. $f(N(u) \times V(H))=0$. In this case, we choose a vertex $u^{\prime} \in N(u)$ and define a function $f^{\prime}$ as $f^{\prime}\left(V\left(H_{u^{\prime}}\right)\right)=f^{\prime}\left(u^{\prime}, v\right)=1, f^{\prime}\left(V\left(H_{u}\right)\right)=f^{\prime}(u, v)=2$ and $f^{\prime}(x, y)=$ $f(x, y)$ for every $x \in V(G) \backslash\left\{u, u^{\prime}\right\}$. As in Case $1, f^{\prime}$ is a $\gamma_{t R}(G \circ H)$-function with $\left|R_{f^{\prime}}\right|<\left|R_{f}\right|$, which is a contradiction.

According to the two cases above, (i) follows. Now, for any $\gamma_{t R}(G \circ H)$-function $f\left(V_{0}, V_{1}, V_{2}\right)$ satisfying (i), we define $R_{f}^{\prime}=\left\{x \in V(G): f\left(V\left(H_{x}\right)\right)=2\right.$ and $V\left(H_{x}\right) \cap$ $\left.V_{2}=\emptyset\right\}$. Let $g\left(V_{0}^{\prime}, V_{1}^{\prime}, V_{2}^{\prime}\right)$ be a $\gamma_{t R}(G \circ H)$-function such that $\left|R_{g}^{\prime}\right|$ is minimum among all $\gamma_{t R}(G \circ H)$-functions satisfying (i). Suppose that there exists $u \in R_{g}^{\prime}$. If there exists $u^{\prime} \in$ $N(u)$ such that, $g\left(V\left(H_{u^{\prime}}\right)\right)=2$, then the function $g^{\prime}$ defined by $g^{\prime}\left(V\left(H_{u}\right)\right)=g^{\prime}(u, v)=$ 1 and $g^{\prime}(x, y)=g(x, y)$ for every $x \in V(G) \backslash\{u\}$, is a TRDF on $G \circ H$ of weight $\omega\left(g^{\prime}\right)<\omega(g)=\gamma_{t R}(G \circ H)$, which is a contradiction. Hence, $g(N(u) \times V(H)) \leq 1$ and we can differentiate the following two cases. 
Case $1^{\prime}$. There exists $u^{\prime} \in N(u)$ such that $g\left(V\left(H_{u^{\prime}}\right)\right)=1$. In this case, we define a function $g^{\prime}$ by $g^{\prime}\left(V\left(H_{u}\right)\right)=g^{\prime}(u, v)=2$ and $g^{\prime}(x, y)=g(x, y)$ for every $x \in V(G) \backslash$ $\{u\}$. Notice that $g^{\prime}$ is a $\gamma_{t R}(G \circ H)$-function satisfying (i) and $\left|R_{g^{\prime}}^{\prime}\right|<\left|R_{g}^{\prime}\right|$, which is a contradiction.

Case 2'. $g(N(u) \times V(H))=0$. We fix $u^{\prime} \in N(u)$. Notice that there exists $u^{\prime \prime} \in$ $N\left(u^{\prime}\right) \backslash\{u\}$, with $V\left(H_{u^{\prime \prime}}\right) \cap V_{2}^{\prime} \neq \emptyset$. Hence, we can define a function $g^{\prime}$ as $g^{\prime}\left(V\left(H_{u^{\prime}}\right)\right)=$ $g^{\prime}\left(u^{\prime}, v\right)=g^{\prime}\left(V\left(H_{u}\right)\right)=g^{\prime}(u, v)=1$ and $g^{\prime}(x, y)=g(x, y)$ for every $x \in V(G) \backslash$ $\left\{u, u^{\prime}\right\}$. As in Case $1^{\prime}, g^{\prime}$ is a $\gamma_{t R}(G \circ H)$-function satisfying (i) and $\left|R_{g^{\prime}}^{\prime}\right|<\left|R_{g}^{\prime}\right|$, which is a contradiction.

According to the two cases above, $R_{g}^{\prime}=\emptyset$, and so there exists a $\gamma_{t R}(G \circ H)$-function $h$ defined as $h\left(V\left(H_{u}\right)\right)=h(u, v)=2$ whenever $g\left(V\left(H_{u}\right)\right)=2$ and $h\left(V\left(H_{u}\right)\right)=$ $g\left(V\left(H_{u}\right)\right)$ otherwise. Therefore, $h$ satisfies (i) and (ii).

Lemma 3.2. Let $G$ be a graph with no isolated vertex and $H$ a nontrivial graph with $\gamma(H)=1$. Let $f\left(V_{0}, V_{1}, V_{2}\right)$ be a $\gamma_{t R}(G \circ H)$-function, $A=\left\{x \in V(G): V\left(H_{x}\right) \cap V_{1} \neq\right.$ $\emptyset\}$ and $B=\left\{x \in V(G): V\left(H_{x}\right) \cap V_{2} \neq \emptyset\right\}$. If $f$ satisfies Lemma 3.1, then $B$ is $a$ dominating set and $A \cup B$ is a total dominating set of $G$.

Proof. Let $f\left(V_{0}, V_{1}, V_{2}\right)$ be a $\gamma_{t R}(G \circ H)$-function which satisfies Lemma 3.1. Let $C=$ $V(G) \backslash(A \cup B)$. Obviously, if $x \in C$, then $V\left(H_{x}\right) \subseteq V_{0}$, which implies that $x$ is adjacent to some vertex in $B$ and, since $H$ is a nontrivial graph and $f$ satisfies Lemma 3.1, if $x \in A$, then there exists $y \in V(H)$ such that $(x, y) \in V_{0}$, and so $x$ is adjacent to some vertex in $B$. Hence, $B$ is a dominating set of $G$. Now, since the subgraph of $G \circ H$ induced by $V_{1} \cup V_{2}$ does not have isolated vertices, the subgraph of $G$ induced by $A \cup B$ does not have isolated vertices, which implies that $A \cup B$ is total dominating set of $G$.

For any graph $G$, let $\mathcal{D}(G)$ be the set of dominating sets of $G$, and $\mathcal{D}_{t}(G)$ the set of total dominating sets of $G$. We now proceed to introduce our main tool, which is the following domination parameter.

$$
\xi(G)=\min \left\{|A|+2|B|: B \in \mathcal{D}(G) \text { and } A \cup B \in \mathcal{D}_{t}(G)\right\} .
$$

We say that an ordered pair $(A, B)$ of subsets of $V(G)$ is a $\xi(G)$-pair if $B \in \mathcal{D}(G)$, $A \cup B \in \mathcal{D}_{t}(G)$ and $\xi(G)=|A|+2|B|$.

Theorem 3.3. For any graph $G$ with no isolated vertex and any nontrivial graph $H$ with $\gamma(H)=1$,

$$
\gamma_{t R}(G \circ H)=\xi(G)
$$

Proof. Let $v$ be a universal vertex of $H$. From any $\xi(G)$-pair $(A, B)$ we define the function $f\left(V_{0}, V_{1}, V_{2}\right)$ as $V_{2}=B \times\{v\}, V_{1}=A \times\{v\}$ and $V_{0}=V(G \circ H) \backslash\left(V_{1} \cup V_{2}\right)$. Since $V_{2}$ is a dominating set of $G \circ H$ and $V_{1} \cup V_{2}$ is a total dominating set of $G \circ H$, we can conclude that $f$ is a TRDF on $G \circ H$. Therefore, $\gamma_{t R}(G \circ H) \leq \omega(f)=\left|V_{1}\right|+2\left|V_{2}\right|=|A|+2|B|=\xi(G)$.

Now, let $f^{\prime}\left(V_{0}^{\prime}, V_{1}^{\prime}, V_{2}^{\prime}\right)$ be a $\gamma_{t R}(G \circ H)$-function which satisfies Lemma 3.1. Let $A=\left\{x \in V(G): f^{\prime}\left(V\left(H_{x}\right)\right)=1\right\}$ and $B=\left\{x \in V(G): f^{\prime}\left(V\left(H_{x}\right)\right)=2\right\}$. By Lemma 3.2, $B$ is a dominating set of $G$ and $A \cup B$ is a total dominating set, which implies that $\xi(G) \leq|A|+2|B|=\left|V_{1}^{\prime}\right|+2\left|V_{2}^{\prime}\right|=\gamma_{t R}(G \circ H)$. Therefore, the result follows. 

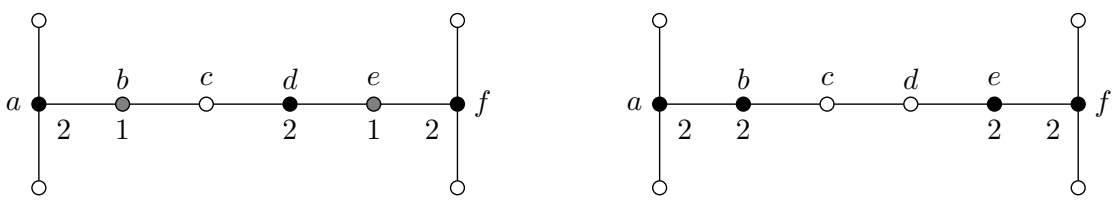

Figure 1: The labels correspond to two different $\gamma_{t R}(G)$-functions $f_{1}\left(V_{0}, V_{1}, V_{2}\right)$, on the left, and $f_{2}\left(W_{0}, W_{1}, W_{2}\right)$, on the right. In this case, $\gamma_{t R}(G)=2 \gamma_{t}(G)=8, V_{2}=\{a, d, f\}$ is a $\gamma(G)$-set and $W_{2}=\{a, b, e, f\}$ is the only $\gamma_{t}(G)$-set.

Let $G$ be the graph shown in Figure 1 and $H$ a nontrivial graph with $\gamma(H)=1$. Notice that $\gamma_{t R}(G \circ H)=\xi(G)=\gamma_{t R}(G)=8$, where $f_{1}\left(V_{0}, V_{1}, V_{2}\right)$ and $f_{2}\left(W_{0}, W_{1}, W_{2}\right)$ are $\gamma_{t R}(G)$-functions for $V_{1}=\{b, e\}, V_{2}=\{a, d, f\}, W_{1}=\emptyset, W_{2}=\{a, b, e, f\}$. Furthermore, both $\left(V_{1}, V_{2}\right)$ and $\left(W_{1}, W_{2}\right)$ are $\xi(G)$-pairs, where $V_{2}$ is a $\gamma(G)$-set and $\left|V_{1}\right|+\left|V_{2}\right|>\gamma_{t}(G)$, while $W_{2}$ is a $\gamma_{t}(G)$-set which does not contain any $\gamma(G)$-set.

The following bounds were given in [5]. In fact the lower bound was stated for any connected non-trivial graph $G$, although it also holds for any graph $G$ with no isolated vertex.

Theorem 3.4 ([5]). For any graph $H$ and any graph $G$ with no isolated vertex,

$$
\gamma_{t R}(G) \leq \gamma_{t R}(G \circ H) \leq 2 \gamma_{t}(G) .
$$

Furthermore, if $\gamma(H)=1$, then

$$
\gamma_{t R}(G \circ H) \leq 3 \gamma(G)
$$

In order to improve some of these bounds, we need to introduce some additional terminology. Given a set $S \subseteq V(G)$, we define

$$
\psi(S)=\min \left\{\left|S^{\prime}\right|: S^{\prime} \subseteq V(G) \backslash S \text { and } S \subseteq N\left(S^{\prime} \cup S\right)\right\} .
$$

We also define the following parameter associated to $G$.

$$
\mu(G)=\min \{\psi(S): S \text { is a } \gamma(G) \text {-set }\} .
$$

It is readily seen that $0 \leq \mu(G) \leq \gamma(G)$. Furthermore, $\mu(G)=0$ if and only if $\gamma_{t}(G)=$ $\gamma(G)$, while $\mu(G)=\gamma(G)$ if and only if for every $\gamma(G)$-set $S$ and every pair of different vertices $x, y \in S$ we have that $N[x] \cap N[y]=\emptyset$, i.e., if and only if every $\gamma(G)$-set is a 2-packing of $G$.

With the notation above in mind, we state the following theorem.

Theorem 3.5. Let $G$ and $H$ be two graphs with no isolated vertex. If $\gamma(H)=1$, then

$$
\max \left\{\gamma_{t R}(G), \gamma_{t}(G)+\gamma(G)\right\} \leq \gamma_{t R}(G \circ H) \leq \min \left\{2 \gamma(G)+\mu(G), 2 \gamma_{t}(G)\right\} .
$$

Proof. Our main tool is Theorem 3.3. For any $\xi(G)$-pair $(A, B)$ we have that $\gamma_{t R}(G \circ H)=$ $\xi(G)=2|B|+|A| \geq|(A \cup B)|+|B| \geq \gamma_{t}(G)+\gamma(G)$.

Now, let $S$ be a $\gamma(G)$-set with $\mu(G)=\psi(S)$ and $S^{\prime} \subseteq V(G) \backslash S$ a set of minimum cardinality among the subsets of $V(G) \backslash S$ satisfying that $S \subseteq N\left(S^{\prime} \cup S\right)$. Since $S \cup S^{\prime}$ is a total dominating set, $\gamma_{t R}(G \circ H)=\xi(G) \leq\left|S \cup S^{\prime}\right|+|S|=2|S|+\left|S^{\prime}\right|=2 \gamma(G)+\mu(G)$.

Finally, by Theorem 3.4, $\gamma_{t R}(G) \leq \gamma_{t R}(G \circ H) \leq 2 \gamma_{t}(G)$, which completes the proof. 
Since $\mu(G) \leq \gamma(G)$, we can conclude that the bound $\gamma_{t R}(G \circ H) \leq 2 \gamma(G)+\mu(G)$ is never worse than the known bound $\gamma_{t R}(G \circ H) \leq 3 \gamma(G)$. In order to see that the upper bounds given by Theorem 3.5 are tight, we take the graph $G$ shown in Figure 1 and any nontrivial graph $H$ with $\gamma(H)=1$. In this case, $\gamma_{t R}(G \circ H)=2 \gamma_{t}(G)=$ $2 \gamma(G)+\mu(G)=8$.

We would point out the following result which is a direct consequence of Theorems 2.2 and 3.5 .

Theorem 3.6. If $G$ is a graph with $\gamma_{t}(G)=\gamma(G)$ and $H$ is a nontrivial graph with $\gamma(H)=1$, then

$$
\gamma_{t R}(G \circ H)=\gamma_{t R}(G)=2 \gamma(G) .
$$

We now proceed to characterize the graphs achieving the lower bounds given by Theorem 3.5 .

Theorem 3.7. Let $G$ and $H$ be two graphs with no isolated vertex. If $\gamma(H)=1$, then the following statements are equivalent.

(i) $\gamma_{t R}(G \circ H)=\gamma_{t R}(G)$.

(ii) There exists a $\gamma_{t R}(G)$-function $f\left(V_{0}, V_{1}, V_{2}\right)$ such that $V_{2}$ is dominating set of $G$.

Proof. If there exists a $\gamma_{t R}(G)$-function $f\left(V_{0}, V_{1}, V_{2}\right)$ such that $V_{2}$ is dominating set of $G$, then $\gamma_{t R}(G \circ H)=\xi(G) \leq\left|V_{1} \cup V_{2}\right|+\left|V_{2}\right|=\left|V_{1}\right|+2\left|V_{2}\right|=\gamma_{t R}(G)$. Since $\gamma_{t R}(G) \leq \gamma_{t R}(G \circ H)$, we conclude that $\gamma_{t R}(G \circ H)=\gamma_{t R}(G)$.

Conversely, assume that $\gamma_{t R}(G \circ H)=\gamma_{t R}(G)$. Let $g\left(V_{0}^{\prime}, V_{1}^{\prime}, V_{2}^{\prime}\right)$ be a $\gamma_{t R}(G \circ H)$ function satisfying Lemma 3.1. Let $A=\left\{x \in V(G): g\left(V\left(H_{x}\right)\right)=1\right\}$ and $B=\{x \in$ $\left.V(G): g\left(V\left(H_{x}\right)\right)=2\right\}$. By Lemma 3.2, $B$ is a dominating set of $G$ and $A \cup B$ is a total dominating set. Hence, we can define a TRDF $h\left(V_{0}^{\prime \prime}, V_{1}^{\prime \prime}, V_{2}^{\prime \prime}\right)$ from $V_{1}^{\prime \prime}=A$ and $V_{2}^{\prime \prime}=B$. Since $\omega(h)=|A|+2|B|=\left|V_{1}^{\prime}\right|+2\left|V_{2}^{\prime}\right|=\gamma_{t R}(G \circ H)=\gamma_{t R}(G)$, we conclude that $h$ is a $\gamma_{t R}(G)$-function where $V_{2}^{\prime \prime}$ is a dominating set, as desired.

The next result gives a characterization for the case $\gamma_{t R}(G \circ H)=\gamma_{t}(G)+\gamma(G)$ whenever $\gamma(H)=1$.

Theorem 3.8. Let $G$ and $H$ be two graphs with no isolated vertex. If $\gamma(H)=1$, then the following statement are equivalent.

(i) $\gamma_{t R}(G \circ H)=\gamma_{t}(G)+\gamma(G)$.

(ii) There exists a $\gamma_{t}(G)$-set that contains some $\gamma(G)$-set.

Proof. If there exists a $\gamma_{t}(G)$-set $X$ which contains a $\gamma(G)$-set $B$, then $\gamma_{t R}(G \circ H)=$ $\xi(G) \leq|X \backslash B|+2|B|=|X|+|B|=\gamma_{t}(G)+\gamma(G)$, and by (i) we conclude that $\gamma_{t R}(G \circ H)=\gamma_{t}(G)+\gamma(G)$.

Conversely, assume that $\gamma_{t R}(G \circ H)=\gamma_{t}(G)+\gamma(G)$ and let $(A, B)$ be a $\xi(G)$-pair. If the total dominating set $A \cup B$ is a $\gamma_{t}(G)$-set, then we are done, as $B$ is a dominating set and from $\gamma_{t}(G)+\gamma(G)=\gamma_{t R}(G \circ H)=\xi(G)=|A|+2|B|=|A \cup B|+|B|=\gamma_{t}(G)+|B|$ we deduce that $B$ is a $\gamma(G)$-set. Suppose to the contrary, that $|A \cup B|>\gamma_{t}(G)$. In such a case, $\gamma_{t}(G)+\gamma(G)=\xi(G)=|A|+2|B| \geq|A \cup B|+|B|>\gamma_{t}(G)+\gamma(G)$, which is a contradiction. Therefore, the result follows.

Figure 2 shows a graph $G$ such that $\gamma_{t R}(G \circ H)=\gamma_{t}(G)+\gamma(G)=7>6=\gamma_{t R}(G)$ for every nontrivial graph $H$ with $\gamma(H)=1$. 


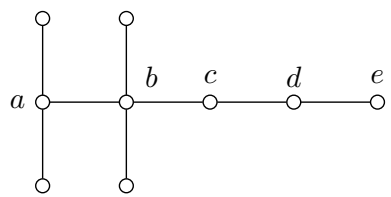

Figure 2: The $\gamma_{t}(G)$-set $D=\{a, b, d, e\}$ contains the $\gamma(G)$-set $S=\{a, b, d\}$.

\section{Small values of $\gamma_{t R}(G \circ H)$}

In this short section we characterize the graphs $G$ and $H$ for which $\gamma_{t R}(G \circ H) \in\{3,4\}$.

Theorem 4.1. For any graph $G$ and $H$ with no isolated vertex, the following statements are equivalent.

(i) $\gamma_{t R}(G \circ H)=3$.

(ii) $\gamma(G)=\gamma(H)=1$.

Proof. If $\gamma_{t R}(G \circ H)=3$, then by Theorem 2.4 we deduce that $\gamma(H)=1$. Moreover, by Theorem 3.5 we have that $3=\gamma_{t R}(G \circ H) \geq \gamma_{t}(G)+\gamma(G) \geq 3$. Hence, $\gamma(G)=1$, as required. Conversely, if $\gamma(G)=\gamma(H)=1$, then by Theorem 3.8 we deduce that $\gamma_{t R}(G \circ H)=3$.

Theorem 4.2. For any graph $G$ and $H$ with no isolated vertex, $\gamma_{t R}(G \circ H)=4$ if and only if one of the following conditions are satisfied.

(i) $\gamma_{t}(G)=2$ and $\gamma(H) \geq 2$.

(ii) $\gamma_{t}(G)=\gamma(G)=2$ and $\gamma(H)=1$.

Proof. We first notice that if conditions (i) or (ii) holds, then by Theorem 2.4 or by Theorem 3.5, respectively, it follows that $\gamma_{t R}(G \circ H)=4$.

Conversely, assume that $\gamma_{t R}(G \circ H)=4$. If $\gamma(H) \geq 2$, then Theorem 2.4 leads to $\gamma_{t}(G)=2$. From now on, we assume that $\gamma(H)=1$. By Theorem 3.8, we have that $4=\gamma_{t R}(G \circ H) \geq \gamma_{t}(G)+\gamma(G)$. Hence, $1 \leq \gamma(G) \leq 2$. If $\gamma(G)=1$, then by Theorem 4.1 we obtain that $\gamma_{t R}(G \circ H)=3$, which is a contradiction. Hence, $\gamma(G)=2$ and so $\gamma_{t}(G)=2$. Therefore, the result follows.

\section{Open problems}

By Theorem 3.3 we learned that, if we want to know the behaviour of $\gamma_{t R}(G \circ H)$ when $\gamma(H)=1$, then it is crucial to obtain the exact value or derive tight bounds on $\xi(G)$. In this sense, the study of $\xi(G)$ is an interesting challenge.

In particular, Theorem 3.5 states that

$$
\max \left\{\gamma_{t R}(G), \gamma_{t}(G)+\gamma(G)\right\} \leq \xi(G) \leq \min \left\{2 \gamma(G)+\mu(G), 2 \gamma_{t}(G)\right\} .
$$

The graphs achieving the equalities $\xi(G)=\gamma_{t R}(G)$ and $\xi(G)=\gamma_{t}(G)+\gamma(G)$ were characterized in Theorems 3.7 and 3.8, respectively. Therefore, the problems of characterizing the graphs achieving the equalities $\xi(G)=2 \gamma_{t}(G)$ and $\xi(G)=2 \gamma(G)+\mu(G)=3 \gamma(G)$ remain open. 


\section{ORCID iDs}

Abel Cabrera Martínez (D) https://orcid.org/0000-0003-2806-4842
Juan Alberto Rodríguez-Velázquez (D) https://orcid.org/0000-0002-9082-7647

\section{References}

[1] H. Abdollahzadeh Ahangar, M. A. Henning, V. Samodivkin and I. G. Yero, Total Roman domination in graphs, Appl. Anal. Discrete Math. 10 (2016), 501-517, doi:10.2298/ aadm160802017a.

[2] B. H. Arriola and S. R. Canoy, Jr., Doubly connected domination in the corona and lexicographic product of graphs, Appl. Math. Sci. 8 (2014), 1521-1533, doi:10.12988/ams.2014. 4136.

[3] A. Cabrera Martínez, S. Cabrera García and J. A. Rodríguez-Velázquez, Double domination in lexicographic product graphs, Discrete Appl. Math. 284 (2020), 290-300, doi:10.1016/j.dam. 2020.03.045.

[4] A. Cabrera Martínez and J. A. Rodríguez-Velázquez, Total protection of lexicographic product graphs, Discuss. Math. Graph Theory (2020), in press, doi:10.7151/dmgt.2318.

[5] N. Campanelli and D. Kuziak, Total Roman domination in the lexicographic product of graphs, Discrete Appl. Math. 263 (2019), 88-95, doi:10.1016/j.dam.2018.06.008.

[6] M. Dettlaff, M. Lemańska, J. A. Rodríguez-Velázquez and R. Zuazua, On the super domination number of lexicographic product graphs, Discrete Appl. Math. 263 (2019), 118-129, doi:10. 1016/j.dam.2018.03.082.

[7] R. Hammack, W. Imrich and S. Klavžar, Handbook of Product Graphs, Discrete Mathematics and its Applications, CRC Press, Boca Raton, FL, 2nd edition, 2011, doi:10.1201/b10959.

[8] T. W. Haynes, S. T. Hedetniemi and P. J. Slater (eds.), Domination in Graphs, Volume 2: Advanced Topics, volume 209 of Monographs and Textbooks in Pure and Applied Mathematics, Marcel Dekker, New York, 1998, doi:10.1201/9781315141428.

[9] T. W. Haynes, S. T. Hedetniemi and P. J. Slater, Fundamentals of Domination in Graphs, volume 208 of Monographs and Textbooks in Pure and Applied Mathematics, Marcel Dekker, New York, 1998, doi:10.1201/9781482246582.

[10] M. A. Henning, A survey of selected recent results on total domination in graphs, Discrete Math. 309 (2009), 32-63, doi:10.1016/j.disc.2007.12.044.

[11] M. A. Henning and A. Yeo, Total Domination in Graphs, Springer Monographs in Mathematics, Springer, New York, 2013, doi:10.1007/978-1-4614-6525-6.

[12] W. Imrich and S. Klavžar, Product Graphs: Structure and Recognition, Wiley-Interscience Series in Discrete Mathematics and Optimization, Wiley-Interscience, New York, 2000.

[13] D. J. Klein and J. A. Rodríguez-Velázquez, Protection of lexicographic product graphs, Discuss. Math. Graph Theory (2019), in press, doi:10.7151/dmgt.2243.

[14] T. Kraner Šumenjak, P. Pavlič and A. Tepeh, On the Roman domination in the lexicographic product of graphs, Discrete Appl. Math. 160 (2012), 2030-2036, doi:10.1016/j.dam.2012.04. 008.

[15] T. Kraner Šumenjak, D. F. Rall and A. Tepeh, Rainbow domination in the lexicographic product of graphs, Discrete Appl. Math. 161 (2013), 2133-2141, doi:10.1016/j.dam.2013.03.011.

[16] R. Laskar, J. Pfaff, S. M. Hedetniemi and S. T. Hedetniemi, On the algorithmic complexity of total domination, SIAM J. Algebraic Discrete Methods 5 (1984), 420-425, doi:10.1137/ 0605040 . 
[17] C.-H. Liu and G. J. Chang, Roman domination on strongly chordal graphs, J. Comb. Optim. 26 (2013), 608-619, doi:10.1007/s10878-012-9482-y.

[18] J. Liu, X. Zhang and J. Meng, Domination in lexicographic product digraphs, Ars Combin. 120 (2015), 23-32.

[19] R. J. Nowakowski and D. F. Rall, Associative graph products and their independence, domination and coloring numbers, Discuss. Math. Graph Theory 16 (1996), 53-79, doi:10.7151/dmgt. 1023.

[20] M. Valveny, H. Pérez-Rosés and J. A. Rodríguez-Velázquez, On the weak Roman domination number of lexicographic product graphs, Discrete Appl. Math. 263 (2019), 257-270, doi:10. 1016/j.dam.2018.03.039.

[21] X. Zhang, J. Liu and J. Meng, Domination in lexicographic product graphs, Ars Combin. 101 (2011), 251-256. 